\title{
Long term dynamics in a mathematical model of HIV-1 infection with delay in different variants of the basic drug therapy model.
}

\author{
Priti Kumar Roy, ${ }^{1}$ Amar Nath Chatterjee ${ }^{\dagger}$, David Greenhalgh ${ }^{2}$ and Qamar \\ J. A. Khan ${ }^{3}$ \\ $1 \dagger$ Centre for Mathematical Biology and Ecology, Department of Mathematics, \\ Jadavpur University, Kolkata - 700032, India. \\ 2 Department of Mathematics and Statistics, University of Strathclyde, Glasgow, UK. \\ 3 Department of Mathematics and Statistics, College of Science, Sultan Qaboos University, \\ Sultanate of Oman.
}

\begin{abstract}
Infection with HIV-1, degrading the human immune system and recent advances of drug therapy to arrest HIV-1 infection, has generated considerable research interest in the area. Sebastian Bonhoeffer et al. [2], introduced a population model representing long term dynamics of HIV infection in response to available drug therapies. We consider a similar type of approximate model incorporating time delay in the process of infection on the healthy $\mathrm{T}$ cells which, in turn, implies inclusion of a similar delay in the process of viral replication. The model is studied both analytically and numerically. We also include a similar delay in the killing rate of infected $\mathrm{CD}^{+} \mathrm{T}$ cells by Cytotoxic $\mathrm{T}$ Lymphocyte (CTL) and in the stimulation of CTL and analyze two resulting models numerically.

The models with no time delay present have two equilibria: one where there is no infection and a non-trivial equilibrium where the infection can persist. If there is no time delay then the non-trivial equilibrium is locally asymptotically stable. Both our analytical results (for the first model) and our numerical results (for all three models) indicate that introduction of a time delay can destabilize the non-trivial equilibrium. The numerical results indicate that such destabilization occurs at realistic time delays and that there is a threshold time delay beneath which the equilibrium with infection present is locally asymptotically stable and above which this equilibrium is unstable and exhibits oscillatory solutions of increasing amplitude.
\end{abstract}

Key words: HIV-1, CD4 ${ }^{+}$T cells, Cytotoxic T-Lymphocyte, Reverse Transcriptase Inhibitor, Asymptotic Stability, Time Delay, Cell Lysis, Time Series Solutions.

\footnotetext{
${ }^{1 *}$ Research is supported by the Government of India, Ministry of Science and Technology, Mathematical Science Office, No. SR/S4/MS: 558/08, Indian National Science Academy and the Royal Society of Edinburgh, UK.

1* E-mail: pritiju@gmail.com

$\dagger$ E-mail: amarbabai@gmail.com

${ }^{2}$ Corresponding Author, E-mail: david.greenhalgh@strath.ac.uk, Fax No.+44 (0)141 548 3345, Ph. No. +44 (0)141 548 3653. Research is supported by the Indian National Science Academy and the Royal Society of Edinburgh, UK.

${ }^{3}$ E-mail: qjalil@squ.edu.om
} 


\section{Introduction:}

Over the last two decades there has been extensive research on the area of HIV-1 infection invading the human immune system. According to the Joint United Nations Programme on HIV/AIDS (UNAIDS), 37 million people worldwide are infected with HIV-1 today of whom 24 million are in developing countries. Though considerable knowledge has been gathered regarding the implications of genetic variation of immune cells, HIV-1 pathogenesis and probable therapies treating the infected individuals, nevertheless controlling HIV-1 infection continues to be a major challenge. HIV-1 infection is associated with an extremely vigorous virus-specific CTL response that declines with disease progression. There are several different HIV-1 vaccinations which include therapeutic vaccination (administered to those who are already infected) and prophylactic vaccination (administered prior to infection) [5], [9].

Anti-retroviral therapy when given to an individual patient makes a portion of the immune cells toxic thereby introducing toxicity in the immune system of the individual. It is thus important to maintain an optimum controlled level of vaccine for an infected individual. Thus use of vaccination at an optimum controlled level for HIV-1 in human infection may emerge that significantly increases the survival time of infected individuals. In particular, immunological escape and subsequent disease progression have been seen in several studies of vaccination therapy [6]-[9]. The main purpose of this study is to develop a mathematical framework that can be used to understand the bearing of drug response on the dynamical behaviour of the lymphocytic immune cells populations.

The main clinical indicators in the follow up of HIV-1 positive patients are both the viral load and the $\mathrm{CD}^{+} \mathrm{T}$ cell count in blood plasma [16]-[19]. It has been observed clinically that patients infected with immunodeficiency virus type-1 (HIV-1), if treated with a combination of inhibitor drugs lamivudine and zidovudine show a 10 to 100 fold reduction of viral load and nearly $25 \%$ increase in the healthy $\mathrm{CD} 4^{+} \mathrm{T}$ cells count. Survival of patients who receive such drugs is observed to be very substantially increased [2]-[6], [9], [13], [15], [17], [18], [30] and [32]. The longer survival is due to consequences of the diminishing rate of infections of the uninfected T cells. This obviously leads to the conjecture that the drug effectively drives the virus to a state of near extinction.

In this paper we propose and analyze a mathematical model of HIV-1 infection to CD4 ${ }^{+}$ $\mathrm{T}$ cells including the inhibitor drug mentioned above. The system responds to the drugstimulation by generating CTL and this CTL in turn attacks the actively infected CD4 ${ }^{+} \mathrm{T}$ cells and kills them. Note that there exists a finite time lag or delay in the process of disease transmission. Also it can be noted that there exists a finite time lag between a $\mathrm{CD} 4^{+} \mathrm{T}$ cell getting actively infected and its subsequent death. Such a realistic time lag has been incorporated in the model under consideration. We have also considered the delay factor in the terms representing killing of virus-producing cells by CTL and in the stimulation of CTL. We analyze the dynamics of such a model in three different cases to understand how the HIV1 infected immune system responds to varied levels of drugs applied under the systematic therapy procedure. The aim is to study and compare the dynamics of the proposed model including delay in three different cases to explore the crucial system parameters and their ranges in order to obtain different theoretical behaviours predicted from the interaction between infectible and infected $\mathrm{CD} 4^{+} \mathrm{T}$ cells and also CTL response against virus infected cells. 


\section{Previous work:}

It is not possible to give a comprehensive review of mathematical models for the internal viral dynamics of HIV. There are too many of these and that is not the purpose of this paper. Instead we give some basic relevant models and their main results. Bonhoeffer et al. [2] consider two models. The first has viral dynamics of only infectible cells $(x)$ and virus-producing cells $(y)$

$$
\begin{aligned}
& \frac{d x}{d t}=\lambda-d x-\beta x y, \\
& \frac{d y}{d t}=\beta x y-a y,
\end{aligned}
$$

where $\lambda$ is the rate of immigration or creation of infectible cells, $d$ is the natural death rate of infectible cells, $a$ is the death rate of virus-producing cells and $\beta$ is the rate of infection of uninfected cells. They examine the effect of reverse transcriptase inhibitors. They calculate $R_{0}$, the basic reproductive number both with and without treatment. $R_{0}$ is defined as the expected number of virus-producing cells caused by a single virus-producing cell entering the disease-free equilibrium (DFE)

$$
R_{0}=\frac{\beta \lambda}{a d}
$$

They show that for $R_{0} \leq 1$ there is only the equilibrium with no virus-producing cells whereas for $R_{0}>1$ there is a unique equilibrium with both infectible cells and virus-producing cells. Simulations were performed to examine the dynamic behaviour of the model.

Later they introduce another variable $z$ for the density of CTL responses against virusinfected cells. The extended model is

$$
\begin{aligned}
& \frac{d x}{d t}=\lambda-d x-\beta x y, \\
& \frac{d y}{d t}=\beta x y-a y-p y z, \\
& \frac{d z}{d t}=k y-b z .
\end{aligned}
$$

Here $p$ is the killing rate of virus-producing cells by CTL, $k$ is the rate of stimulation of CTL and $b$ is the per capita death rate of CTL. For this model $R_{0}$ is still given by (1.2).

In another paper Bonhoeffer et al. [3] examine the dynamics of uninfected cells $(x)$, infected cells $(y)$ and virus $(v)$. The equations are:

$$
\begin{aligned}
& \frac{d x}{d t}=\lambda-d x-\beta x v, \\
& \frac{d y}{d t}=\beta x v-a y, \\
& \frac{d v}{d t}=k y-b v .
\end{aligned}
$$

Here $k$ is interpreted as the rate of production of virus by infected cells and $b$ is the per particle death rate of the virus. They show that the basic reproductive number, here defined as the expected number of secondary virus particles produced by a single new virus particle entering a population of uninfected cells at equilibrium with neither infected cells nor virus present is

$$
R_{0}=\frac{\beta \lambda k}{a b d} .
$$

For the above model two equilibria existed. The virus-free equilibrium (VFE) was

$$
x=\hat{x}=\frac{\lambda}{d}, y=\hat{y}=0 \text { and } v=\hat{v}=0
$$


and the endemic equilibrium was given by

$$
x=x^{*}=\frac{a b}{\beta k}, y=y^{*}=\frac{\left(R_{0}-1\right) d b}{\beta k} \text { and } v=v^{*}=\frac{\left(R_{0}-1\right) d}{b} .
$$

They went on to include the dynamics of resistant strains into the basic model. Our models are based on the extended model of Bonhoeffer et al. [2] but we introduce time delays into the model as described later in the paper.

Dalal et al. [8] introduce treatment with highly active antiretroviral therapy (HAART) into the above model. They propose the following three dimensional model to describe the viral dynamics in the presence of HIV-1 infection and HAART

$$
\begin{aligned}
& \frac{d x}{d t}=\lambda-d x-(1-\gamma) \beta x v \\
& \frac{d y}{d t}=(1-\gamma) \beta x v-a y \\
& \frac{d z}{d t}=(1-\eta) N a y-b v-(1-\gamma) \beta x v
\end{aligned}
$$

$1-\gamma$ is the reverse transcriptase inhibitor drug effect and $1-\eta$ is the protease inhibitor drug effect. $a$ is the per capita death rate of infected cells and $N$ is the average number of infective virus particles produced by an infected cell in the absence of HAART during its entire infectious lifetime. Dalal et al. show that this model has a unique virus-free equilibrium (VFE) which is globally asymptotically stable (GAS) provided that the basic reproductive number $R_{0}$ is less than one where

$$
R_{0}=\frac{(1-\gamma) \beta \lambda((1-\eta) N-1)}{d b}
$$

Tuckwell and Wan [38] analysed a functionally similar model but without HAART, corresponding to $\gamma=\eta=0$ in Dalal et al.'s model. By suitable redefinition of parameters in Tuckwell and Wan's model we deduce that for $R_{0}>1$ in Dalal et al.'s model the VFE is unstable and there is a unique non-trivial equilibrium with infected cells and virus particles which is locally asymptotically stable (LAS).

Wang et al. [39] consider the extended model (1.3) of Bonhoeffer et al. [2]. They introduce a time delay into the immune response so that the term $k y$ in the third equation becomes $k y(t-\tau)$. They perform an equilibrium and stability analysis and simulations to confirm the results. They show that the infection-free equilibrium is GAS for $R_{0}<1$. For $R_{0}>1$ complicated dynamic behaviour occurs as the time delay $\tau$ increases if the lytic component $p$ is larger than a given critical value. Moreover complex dynamic behaviour ensues if the birth rate $\lambda$ of susceptible cells exceeds a critical value. Stability switches, periodic solutions and chaotic solutions can be observed via computer simulations.

Zhu and Zou [44] consider a model based on the extended model (1.3) of Bonhoeffer et al. [2] with a time delay in both the infection term and the immune response term. They examine the stability using analytical results and simulations. Their model differs from our model in two ways. First in their model a susceptible cell which is contacted by an infected cell enters an incubating class, whereas in our model such cells remain in the susceptible class until they either die or a time $\tau$ later enter the infected class. Secondly they consider an additional time delay in the term representing killing of virus-producing cells by CTL. They derive an expression for the basic reproduction number $R_{0}$ for their model and show that for $R_{0}<1$ the infection-free equilibrium is GAS, but for $R_{0}>1$ it is unstable. In the latter case if $R_{0}>1$ there is a unique equilibrium with disease present which is always LAS. Simulations confirm these results. 


\section{Formulation of the mathematical model:}

Whenever a HIV-1 infected patient is subjected to an RTI (Reverse Transcriptase Inhibitor) drug, the virus replication within the virus-producing $\mathrm{T}$ cells faces a reduction. Such an effect may be incorporated in the two variable simple virus dynamics model (1.1) by reducing the numerical value of the parameter $\beta$ (the infection transmission coefficient). However mere reduction of $\beta$ in the basic virus dynamics model fails to explain the strong suppression of equilibrium virus load during long-term drug therapy. Therefore it is helpful to include another variable in the basic virus dynamics model (1.1) in order to make the long-term response of the model more realistic. This gives us the extended model (1.3) of Bonhoeffer et al. [2] which can be regarded as our basic drug therapy model.

In the basic model (1.3) including CTL response of an HIV-1 infected system the process of interaction between the infected $\mathrm{CD} 4^{+} \mathrm{T}$ cells and infectible $\mathrm{CD} 4^{+} \mathrm{T}$ cells, through which transmission of the disease takes place, is considered as instantaneous. However the natural disease transmission process requires finite time. An infectible CD4 ${ }^{+} \mathrm{T}$ cell becomes the target of an already infected cell and is transformed to the infected class following a time consuming transmission process. This means that a time lag or delay exists in the process of disease transmission. Thus the model equation can be rewritten in the form

$$
\begin{aligned}
& \frac{d x}{d t}=\lambda-d x-\beta x y, \\
& \frac{d y}{d t}=\beta^{\prime} \int_{-\infty}^{t} x(u) y(u) F(t-u) d u-a y-p y z \\
& \frac{d z}{d t}=k y-b z .
\end{aligned}
$$

Here we assume that the cells which are productively infectious at time $t$, were infected $u$ time units ago where $u$ is distributed according to a probability distribution $F(u)$, known as the delay kernel and defined as $F(u)=\frac{\alpha^{n+1} u^{n}}{n !} e^{-\alpha u}$, where $\alpha>0$ is constant and $n$ is the order of delay [23] and the average delay is defined by $\tau=\int_{0}^{\infty} u F(u) d u=\frac{n+1}{\alpha}$. Here the kernel takes the form $F(u)=\delta(u-\tau)$ where $\tau \geq 0$ is constant. Then the system becomes the following delay differential equation with delay $\tau[7]$ :

$$
\begin{aligned}
& \frac{d x}{d t}=\lambda-d x-\beta x y, \\
& \frac{d y}{d t}=\beta^{\prime} x(t-\tau) y(t-\tau)-a y-p y z, \\
& \frac{d z}{d t}=k y-b z .
\end{aligned}
$$

Here we also consider that the contact process between the uninfected and virus-producing cells is not instantaneous. Thus we include a delay, similar to the disease transmission term, in the rate of contact term in the first equation in (1.9). Thus the equations take the form

$$
\begin{aligned}
& \frac{d x}{d t}=\lambda-d x-\beta x(t-\tau) y(t-\tau) P(t, \tau), \\
& \frac{d y}{d t}=\beta x(t-\tau) y(t-\tau) P(t, \tau)-a y-p y z, \\
& \frac{d z}{d t}=k y-b z,
\end{aligned}
$$

with initial conditions $x(t-\tau)=0, y(t-\tau)=0$ for $t-\tau<0, x(0)=x_{0}, y(0)=y_{0}$ and $z(0)=z_{0}$. Here $P(t, \tau)$ is the probability that a susceptible x-cell survives $[t-\tau, t)$.

In fact let

$$
P(\eta)=P(\text { susceptible } \mathbf{x}-\text { cell survives }[\eta-\tau, \eta))
$$

then 


$$
\begin{aligned}
P(\eta) & =\exp \left[-\int_{\eta-\tau}^{\eta}\left(d+\frac{\beta x(\xi-\tau) y(\xi-\tau) P(\xi)}{x(\xi)}\right) d \xi\right] \\
& =\exp \left[-d \tau-\int_{\eta-\tau}^{\eta} \frac{\beta x(\xi-\tau) y(\xi-\tau) P(\xi)}{x(\xi)} d \xi\right]
\end{aligned}
$$

and $P(0)=\exp (-d \tau)$. Equivalently

$$
\frac{1}{P(\eta)} \frac{d P}{d \eta}=\frac{\beta x(\eta-\tau) y(\eta-\tau) P(\eta)}{x(\eta)}-\frac{\beta x(\eta-2 \tau) y(\eta-2 \tau) P(\eta-\tau)}{x(\eta-\tau)}
$$

with $P(0)=\exp (-d \tau)$. In fact $P(\eta)=\exp (-d \tau)$ for $\eta \leq \tau$.

These equations are very complicated to analyze. If the time delay is relatively small they can be approximated by the simpler set of equations:

$$
\begin{aligned}
& \frac{d x}{d t}=\lambda-d x-\beta x(t-\tau) y(t-\tau), \\
& \frac{d y}{d t}=\beta x(t-\tau) y(t-\tau)-a y-p y z, \\
& \frac{d z}{d t}=k y-b z
\end{aligned}
$$

with the same initial conditions as (1.10).

Thus we are effectively assuming for simplicity that susceptible cells which become infected at time $t-\tau$ all survive up to time $\tau$. This approximation is similar to the approximation made by many authors when the uninfected $\mathrm{T}$ cells enter an incubating class upon contact with virus-producing $\mathrm{T}$ cells and become virus-producing $\mathrm{T}$ cells $\tau$ time units later. A model which takes into account death of contacted cells during the incubation period is used by Culshaw et al. [7] and Zhu and Zou [44]. However these deaths are ignored as an approximation by other authors (Shi et al. [35], Li and Ma [21], McCluskey [26] and Li et al. [22]). Because in our model the contacted cells remain in the susceptible class rather than progressing to the incubating class, as well as deaths we must also take account of those contacted $\mathrm{T}$ cells which exit the susceptible class during the time interval $[t-\tau, t)$ because of also previously being contacted in the time interval $[t-2 \tau, t-\tau)$, as described in equations (1.10). However the idea of our approximation is similar.

Yang et al. [42] propose a delay differential equation model which uses exactly the same approximation as we do, with exactly the same contact terms, except that the infection is initiated by direct contact with the virus, rather than direct contact with the virus-producing T cells. A similar idea is used in another context related to HIV infection by Bachar and Dorfmayr [1]. The same idea behind the approximation that we have used has been used by many other authors when modelling time delays. In this paper we study the behaviour of the approximate model (1.11) as the full model (1.10) is too complicated.

\section{Local stability analysis:}

The right hand side of the equation (1.11) is a smooth function of $x, y, z$ (the variables) and the parameters, as long as the quantities are non-negative, so local existence and uniqueness properties hold in the positive octant. The model equations (1.11) have equilibria $E_{1}\left(\frac{\lambda}{d}, 0,0\right)$ and $E^{*}\left(x^{*}, y^{*}, z^{*}\right)$ where,

$$
x^{*}=\frac{(a b \beta-d k p)+\sqrt{(a b \beta-d k p)^{2}+4 \beta^{2} b p k \lambda}}{2 b \beta^{2}}, \quad y^{*}=\frac{\lambda-d x^{*}}{\beta x^{*}} \text { and } z^{*}=\frac{\beta x^{*}-a}{p} .
$$


Note that $E^{*}$ is feasible and non-trivial if and only if $\frac{a}{\beta}<\frac{\lambda}{d}$. Here we are interested to investigate the local stability of the interior equilibrium $E^{*}$ of the delay-induced system (1.11).

Let $\tilde{x}(t)=x(t)-x^{*}, \tilde{y}(t)=y(t)-y^{*}, \tilde{z}(t)=z(t)-z^{*}$ be the perturbed variables. The linearized form of the system $(1.11)$ at $E^{*}\left(x^{*}, y^{*}, z^{*}\right)$ is given by

$$
\begin{aligned}
& \frac{d \tilde{x}}{d t}=-d \tilde{x}-\beta \tilde{x}(t-\tau) y^{*}-\beta \tilde{y}(t-\tau) x^{*}, \\
& \frac{d \tilde{y}}{d t}=\beta \tilde{x}(t-\tau) y^{*}+\beta \tilde{y}(t-\tau) x^{*}-a \tilde{y}-p \tilde{y} z^{*}-p \tilde{z} y^{*}, \\
& \frac{d \tilde{z}}{d t}=k \tilde{y}-b \tilde{z} .
\end{aligned}
$$

The characteristic equation of system (2.1) is given by

$$
\rho^{3}+\left(A+\beta B e^{-\rho \tau}\right) \rho^{2}+\left(C+\beta D e^{-\rho \tau}\right) \rho+\left(E+\beta F e^{-\rho \tau}\right)=0,
$$

where,

$$
\begin{aligned}
& A=a+b+d+p z^{*}(>0) \\
& B=y^{*}-x^{*} \\
& C=d\left(a+b+p z^{*}\right)+a b+b p z^{*}+p k y^{*}(>0), \\
& D=a y^{*}+b y^{*}+p y^{*} z^{*}-d x^{*}-b x^{*} \\
& E=d\left(a b+b p z^{*}+p k y^{*}\right) \\
& F=y^{*}\left(p k y^{*}+b \beta x^{*}\right)-d b x^{*}
\end{aligned}
$$

Now to determine the nature of the stability, we require the sign of the real parts of the roots of the system (2.2).

Let,

$$
\Phi(\rho, \tau)=\rho^{3}+\left(A+\beta B e^{-\rho \tau}\right) \rho^{2}+\left(C+\beta D e^{-\rho \tau}\right) \rho+\left(E+\beta F e^{-\rho \tau}\right) .
$$

For $\tau=0$, i.e for the non-delayed system

$$
\Phi(\rho, 0)=\rho^{3}+(A+\beta B) \rho^{2}+(C+\beta D) \rho+(E+\beta F)=0 .
$$

\section{Theorem 2.1:}

For $\tau=0$ the unique non-trivial equilibrium is LAS.

\section{Proof:}

To show that the Routh-Hurwitz conditions [25] are satisfied we need to show that $A+$ $\beta B>0, E+\beta F>0$ and $(A+\beta B)(C+\beta D)>E+\beta F$.

(i) $A+\beta B=a+b+d+p z^{*}+\beta\left(y^{*}-x^{*}\right)$, $=b+d+\beta y^{*}>0$.

(ii) $C+\beta D=d\left(a+b+p z^{*}\right)+a b+b p z^{*}+p k y^{*}+\beta\left(a y^{*}+b y^{*}+p y^{*} z^{*}-d x^{*}-b x^{*}\right)$, $=d b+p k y^{*}+\beta\left(b y^{*}+\beta x^{*} y^{*}\right)>0$.

(iii) $E+\beta F=d\left(a b+b p z^{*}+p k y^{*}\right)+\beta y^{*}\left(p k y^{*}+b \beta x^{*}\right)-d b \beta x^{*}$, $=d p k y^{*}+\beta y^{*}\left(p k y^{*}+b \beta x^{*}\right)$, $>0$.

(iv) Using the above expressions it is straightforward to show that Theorem 2.1 is true. 
Substituting $\rho=u(\tau)+i v(\tau)$ in $(2.2)$ and separating real and imaginary parts we obtain the following transcendental equations

$$
\begin{aligned}
& u^{3}-3 u v^{2}+\left(u^{2}-v^{2}\right)\left(A+\beta B e^{-u \tau} \cos v \tau\right)+2 u v \beta B e^{-u \tau} \sin v \tau+C u \\
& +u \beta D e^{-u \tau} \cos v \tau+v \beta D e^{-u \tau} \sin v \tau+E+\beta F e^{-u \tau} \cos v \tau=0 \\
& 3 u^{2} v-v^{3}+2 u v A+2 u v \beta B e^{-u \tau} \cos v \tau-\left(u^{2}-v^{2}\right) \beta B e^{-u \tau} \sin v \tau+C v \\
& +v \beta D e^{-u \tau} \cos v \tau-\beta(u D+F) e^{-u \tau} \sin v \tau=0
\end{aligned}
$$

\section{Sufficient Conditions for Nonexistence of Delay Induced Instability:}

To find the conditions for nonexistence of delay induced instability, we now use the following theorem [12].

Theorem 3.1: A set of necessary and sufficient conditions for the equilibrium $E^{*}$ to be asymptotically stable for all $\tau \geq 0$ is the following:

(i) The real parts of all the roots of $\Phi(\rho, 0)=0$ are negative.

(ii) For all real $v$ and $\tau \geq 0, \Phi(i v, \tau) \neq 0$, where $i=\sqrt{-1}$.

Proof: Here $\Phi(\rho, 0)=0$ has roots whose real parts are negative. Now for $v=0$

$$
\Phi(0, \tau)=E+\beta F \neq 0
$$

and for $v \neq 0$

$$
\Phi(i v, \tau)=-i v^{3}-\left(A+\beta B e^{-i v \tau}\right) v^{2}+i v\left(C+\beta D e^{-i v \tau}\right)+\left(E+\beta F e^{-i v \tau}\right)=0
$$

Separating real and imaginary parts we get,

$$
A v^{2}-E=v \beta D \sin v \tau+\beta\left(F-B v^{2}\right) \cos v \tau
$$

and

$$
-v^{3}+C v=-v \beta D \cos v \tau+\beta\left(F-B v^{2}\right) \sin v \tau .
$$

Squaring and adding the above two equations, we get

$$
\left(A v^{2}-E\right)^{2}+\left(-v^{3}+C v\right)^{2}=v^{2} \beta^{2} D^{2}+\beta^{2}\left(F-B v^{2}\right)^{2} .
$$

Therefore a sufficient condition for the non existence of a real number $v$ satisfying $\Phi(i v, \tau)=0$ can now be obtained from (3.5) as

$$
v^{6}+\left(A^{2}-2 C-\beta^{2} B^{2}\right) v^{4}+\left(C^{2}-2 A E-\beta^{2} D^{2}+2 B F \beta^{2}\right) v^{2}+E^{2}-\beta^{2} F^{2}>0
$$

for all real $v$.

We can write this inequality in the form of

$$
v^{6}+P v^{4}+Q v^{2}+R>0
$$


where,

$$
\begin{aligned}
& P=A^{2}-2 C-B^{2} \beta^{2}, \\
& Q=C^{2}-2 A E-D^{2} \beta^{2}+2 B F \beta^{2}, \\
& R=E^{2}-\beta^{2} F^{2} .
\end{aligned}
$$

Therefore conditions (i) and (ii) of Theorem 3.1 are satisfied if (3.6) holds.

\section{Criterion for Preservation of Stability, Instability and Bifurcation Results:}

Let us consider $\rho$ and hence $u$ and $v$ as functions of $\tau$. We are interested in the change of stability of $E^{*}$ which will occur at the values of $\tau$ for which $u=0$ and $v \neq 0$. Let $\hat{\tau}$ be such that for which $u(\hat{\tau})=0$ and $v(\hat{\tau})=\hat{v} \neq 0$. Then equations (2.6) and (2.7) become

$$
\begin{gathered}
-A \hat{v}^{2}-\hat{v}^{2} \beta B \cos \hat{v} \hat{\tau}+\hat{v} \beta D \sin \hat{v} \hat{\tau}+\beta F \cos \hat{v} \hat{\tau}+E=0 \\
-\hat{v}^{3}+\hat{v}^{2} \beta B \sin \hat{v} \hat{\tau}+C \hat{v}+\hat{v} \beta D \cos \hat{v} \hat{\tau}-\beta F \sin \hat{v} \hat{\tau}=0
\end{gathered}
$$

Now eliminating $\hat{\tau}$ we get,

$$
\hat{v}^{6}+\left(A^{2}-2 C-\beta^{2} B^{2}\right) \hat{v}^{4}+\left(C^{2}-\beta^{2} D^{2}-2 A E+2 B F \beta^{2}\right) \hat{v}^{2}+\left(E^{2}-\beta^{2} F^{2}\right)=0 .
$$

To analyze the change in the behaviour of the stability of $E^{*}$ with respect to $\tau$, we examine the sign of $\frac{d u}{d \tau}$ as $u$ crosses zero. If this derivative is positive (negative) then clearly a stabilization (destabilization) cannot take place at that value of $\hat{\tau}$. We differentiate equations (2.6) and (2.7) w.r.t. $\tau$, then setting $\tau=\hat{\tau}, u=0$ and $v=\hat{v}$ we get,

$$
\begin{array}{r}
X \frac{d u}{d \tau}(\hat{\tau})+Y \frac{d v}{d \tau}(\hat{\tau})=g, \\
-Y \frac{d u}{d \tau}(\hat{\tau})+X \frac{d v}{d \tau}(\hat{\tau})=h
\end{array}
$$

where,

$$
\begin{aligned}
& X=-3 \hat{v}^{2}+C+D \beta \cos \hat{v} \hat{\tau}+2 \beta \hat{v} B \sin \hat{v} \hat{\tau}+\hat{\tau}\left[\left(\beta \hat{v}^{2} B-\beta F\right) \cos \hat{v} \hat{\tau}-\beta \hat{v} D \sin \hat{v} \hat{\tau}\right], \\
& Y=-2 A \hat{v}+\beta D \sin \hat{v} \hat{\tau}-2 \hat{v} \beta B \cos \hat{v} \hat{\tau}+\hat{\tau}\left[\left(\beta \hat{v}^{2} B-\beta F\right) \sin \hat{v} \hat{\tau}+\hat{v} \beta D \cos \hat{v} \hat{\tau}\right], \\
& g=-\left[\left(\beta \hat{v}^{2} B-\beta F\right) \sin \hat{v} \hat{\tau}+\hat{v} \beta D \cos \hat{v} \hat{\tau}\right] \hat{v} \\
& h=-\left[\left(\beta \hat{v}^{2} B-\beta F\right) \cos \hat{v} \hat{\tau}-\hat{v} \beta D \sin \hat{v} \hat{\tau}\right] \hat{v} .
\end{aligned}
$$

Solving (4.4) we get,

$$
\frac{d u}{d \tau}(\hat{\tau})=\frac{g X-h Y}{X^{2}+Y^{2}}
$$

$\frac{d u}{d \tau}(\hat{\tau})$ has the same sign as $g X-h Y$.

After simplification and solving (4.1) and (4.2) we get,

$$
g X-h Y=\hat{v}^{2}\left[3 \hat{v}^{4}+2\left(A^{2}-2 C-B^{2} \beta^{2}\right) \hat{v}^{2}+\left(C^{2}-\beta^{2} D^{2}-2 A E+2 \beta^{2} B F\right)\right] .
$$

Let,

$$
F(z)=z^{3}+P_{1} z^{2}+P_{2} z+P_{3}
$$


where

$$
P_{1}=A^{2}-2 C-\beta^{2} B^{2}, P_{2}=C^{2}-\beta^{2} D^{2}-2 A E+2 \beta^{2} B F, P_{3}=E^{2}-\beta^{2} F^{2},
$$

which is the left hand side of the equation (4.3) with $\hat{v}^{2}=z$.

Therefore,

$$
F\left(\hat{v}^{2}\right)=0
$$

Now

$$
\begin{aligned}
& \frac{d F}{d z}\left(\hat{v}^{2}\right)=3 \hat{v}^{4}+2 P_{1} \hat{v}^{2}+P_{2}=\frac{g X-h Y}{\hat{v}^{2}} . \\
\Rightarrow & \frac{d F}{d z}\left(\hat{v}^{2}\right)=\frac{X^{2}+Y^{2}}{\hat{v}^{2}} \cdot \frac{d u}{d \tau}(\hat{\tau}) \\
\Rightarrow & \frac{d u}{d \tau}(\hat{\tau})=\frac{\hat{v}^{2}}{X^{2}+Y^{2}} \cdot \frac{d F}{d z}\left(\hat{v}^{2}\right) .
\end{aligned}
$$

Hence if $z$ is a positive simple root of $F(z)=0$ and $z=\hat{v}^{2}$ then $\frac{d u}{d \tau}(\hat{\tau}) \neq 0$ and at $\hat{\tau}, u=0, v=\hat{v}$ and the corresponding root of the characteristic equation crosses the imaginary axis transversally. Thus by the Hopf Bifurcation Theorem [24] limit cycles arise and disappear at those values of $\hat{\tau}$.

$F(z)$ has zero, one, two or three positive real roots. We have the following theorem:

\section{Theorem 4.1:}

(i) If $F(z)$ has no positive real roots then the non-trivial equilibrium $E^{*}$ is always LAS.

(ii) If $F(z)$ has one positive simple real root $\hat{v}$ then $F^{\prime}(\hat{v})>0$. In this case there exists $\hat{\tau}_{1}>0$ such that the non-trivial equilibrium is LAS for $\tau \in\left[0, \hat{\tau}_{1}\right)$ and unstable for $\tau>\hat{\tau}_{1}$. $\hat{\tau}_{1}$ is the unique root in $(0,2 \pi / \hat{v}]$ of the equations:

$$
\begin{aligned}
\sin \hat{v} \tau & =\frac{\left(A \hat{v}^{2}-E\right) \hat{v} D+\left(\hat{v}^{3}-C \hat{v}\right)\left(\hat{v}^{2} B-F\right)}{\beta\left[(\hat{v} D)^{2}+\left(\hat{v}^{2} B-F\right)^{2}\right]} \\
\cos \hat{v} \tau & =\frac{\left(\hat{v}^{3}-C \hat{v}\right) \hat{v} D-\left(A \hat{v}^{2}-E\right)\left(\hat{v}^{2} B-F\right)}{\beta\left[(\hat{v} D)^{2}+\left(\hat{v}^{2} B-F\right)^{2}\right]} .
\end{aligned}
$$

As $\tau$ passes through $\hat{\tau}_{1}$ small amplitude periodic solutions arise by Hopf bifurcation.

(iii) If $F(z)$ has two or three positive simple roots then at the largest of these $F^{\prime}(v)>0$ and at the second largest $F^{\prime}(v)<0$. In this case there exists a sequence $\hat{\tau}_{0}=0, \hat{\tau}_{1}, \hat{\tau}_{2}, \hat{\tau}_{3}, \ldots$ such that for $\tau \in\left(\hat{\tau}_{2 j}, \hat{\tau}_{2 j+1}\right), j=0,1,2,3, \ldots$ the non-trivial equilibrium is LAS, and for $\tau \in\left(\hat{\tau}_{2 j+1}, \hat{\tau}_{2 j+2}\right), j=0,1,2,3, \ldots$ the non-trivial equilibrium is unstable. $\hat{\tau}_{2 j+1}$ is the next root of (4.10) after $\hat{\tau}_{2 j}$ corresponding to $\hat{v}$ a positive root of $F(v)=0$ where $F^{\prime}(v)>0$, and $\hat{\tau}_{2 j+2}$ is the next root of (4.10) after $\hat{\tau}_{2 j+1}$ corresponding to a positive root of $F(v)=0$ where $F^{\prime}(v)<0$. As $\tau$ passes through each $\tau_{2 j+1}$ small periodic solutions arise which are caused by Hopf bifurcation and disappear by backwards Hopf bifurcation as $\tau$ passes through $\hat{\tau}_{2 j+2}$.

(iv) If $F(z)$ has a repeated positive real root it is not possible to say as much.

(a) Suppose that $\hat{\tau}_{1}$ the first root of $\hat{\tau}$ given by (4.10) corresponding to a real positive root $\hat{v}$ of $F(v)$ is associated with a simple root $\hat{v}$ with $F^{\prime}(\hat{v})>0$. Then the non-trivial equilibrium is again LAS for $\tau \in\left[0, \hat{\tau}_{1}\right)$ and unstable for $\tau \in\left(\hat{\tau}_{1}, \hat{\tau}^{*}\right)$, where $\hat{\tau}^{*}$ is the next value of $\hat{\tau}$ given by (4.10) after $\hat{\tau}_{1}$ corresponding to a real positive repeated root $\hat{v}$ of $F(v)=0$. It is not possible to say anything about the system for $\tau \geq \tau^{*}$. 
(b) Suppose that $\hat{\tau}_{1}$, the first value of $\hat{\tau}$ given by (4.10) corresponding to a positive real root $\hat{v}$ of $F(v)=0$, corresponds to either a repeated root or a simple root with $F^{\prime}(\hat{v})<0$. Then the non-trivial equilibrium is LAS for $\tau \in\left[0, \hat{\tau}_{1}^{*}\right)$, where $\hat{\tau}_{1}^{*}$ is the first value of $\hat{\tau}$ given by (4.10) corresponding to a repeated positive root $\hat{v}$ of $F(v)=0$. Again it is not possible to say anything about the system for $\tau \geq \tau_{1}^{*}$.

This completes our analytical study of our basic mathematical model (1.11). In the next section we shall explore this model and two other similar models numerically.

\section{Numerical Simulations: Results and Discussions:}

Table. 1 Variables and parameters used in the models.

\begin{tabular}{|c|c|c|}
\hline Parameters & Definition & Default Values Assigned \\
\hline $\bar{\lambda}$ & Constant production rate of CD $4^{+} \mathrm{T}$ cells & $10.0 \mathrm{~mm}^{-3}$ day $^{-1}[31]$ \\
\hline$d$ & Death rate of susceptible CD $4^{+} \mathrm{T}$ cells & 0.01 day $^{-1}[31]$ \\
\hline$\beta$ & Rate of contact between $x$ and $y$ & $0.002 \mathrm{~mm}^{-3}$ day $^{-1}[41]$ \\
\hline$a$ & Death rate of virus-producing cells & 0.24 day $^{-1}[31]$ \\
\hline$k$ & Rate of stimulation of CTL & 0.2 day $^{-1}[40][41]$ \\
\hline$b$ & Death rate of CTL & 0.02 day $^{-1}[2]$ \\
\hline$p$ & Killing rate of virus-producing cells by CTL & $0.001-0.005 \mathrm{~mm}^{-3} \mathrm{day}^{-1}[41]$ \\
\hline$\tau$ & Incubation delay & $1.0-15.0$ days $[42]$ \\
\hline
\end{tabular}

Numerical simulation of the model (1.11) is done with the basic model parameters set to their standard values as in Table 1 . The variation of the parameter $p$, the killing rate of virus-producing cells by CTL is restricted by the condition $\frac{p k}{b} \sim 0.01-0.05 \mathrm{~mm}^{-3}$ day $^{-1}[2]$. The parameters $k$ and $b$ are as mentioned in the Table 1. From the existence and stability analysis for the non-delayed and delayed system $p$ seems to be an important parameter.

In Figure 1 we plot the time series solutions of the model variables corresponding to uninfected T cells, infected T cells and CTL densities for different values of the delay factor $\tau$. All the other model parameters are chosen to assume their standard values as in Table 1. The starting point is obtained by perturbing $x$ from the non-trivial equilibrium value $E^{*}$ given by $\left(x^{*}, y^{*}, z^{*}\right)=(212.5947,18.5189,185.1893)$. Figure 1(a) (Figure 1, top left) has initial condition $x(\theta)=280.0, y(\theta)=18.5189$ for $\theta \in(-\tau, 0]$ and $z(0)=185.1893$. Figures 1(b)-(d) (Figure 1, top right, bottom left and bottom right, respectively) have initial conditions $x(\theta)=240.0, y(\theta)=18.5189$ for $\theta \in(-\tau, 0]$ and $z(0)=185.1893$. Figure 1 (a) has $\tau=1$ day, Figure 1(b) has $\tau=3$ days, Figure 1(c) has $\tau=6.2$ days and Figure $1(\mathrm{~d})$ has $\tau=8$ days. In this and the other numerical simulations of our models which we shall present, the initial conditions for $x$ and $y$ have been taken to be constant in $(-\tau, 0]$, with $x$ perturbed slightly from its non-trivial equilibrium value at $E^{*}$. We have done this because our main interest is the stability of the non-trivial equilibrium $E^{*}$.

We observe that introducing and then increasing the delay makes the oscillations in the solution persist for longer. For the parameter values used $F(z)$ has one positive simple real root and the corresponding value of $\hat{\tau}_{1}$ is 6.2 days. Our simulations are consistent with the theoretical results. When $\tau=0$ days the non-trivial equilibrium $E^{*}$ is LAS. Increasing the time delay $\tau$ makes the initial oscillations persist for longer, until we pass through the critical value $\hat{\tau}_{1}=6.2$ days (Figure $1(\mathrm{c})$ ) when there are regular cyclic oscillations. As $\tau$ increases beyond $\hat{\tau}_{1}$, the non-trivial equilibrium $E^{*}$ becomes unstable and the oscillatory solutions 

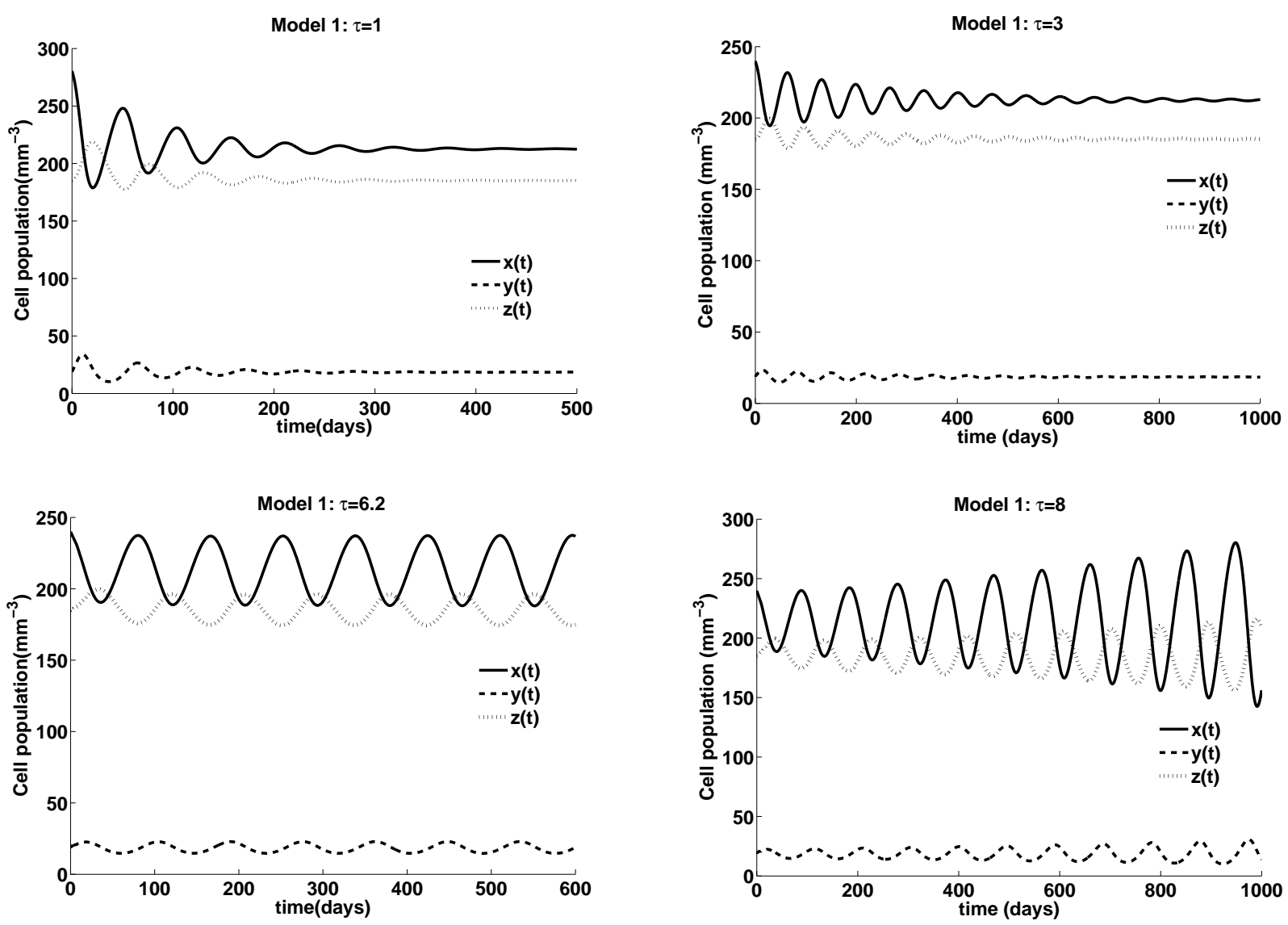

Figure 1: Time series solutions of model variables for different values of $\tau$ with perturbation. The model parameters are as in Table 1. $p=0.001 \mathrm{~mm}^{-3}$ day $^{-1}$.

increase in amplitude until they reach values of the susceptible and infected T cells and CTL where the approximate model becomes unrealistic.

Other simulations (not presented) indicate that as $p$ increases the amplitude of oscillations in the solutions diminishes and reduces the timespan of the persistence of the solutions. The results seem to signify that there is a competition between the delay factor $\tau$ and the killing rate of infected T cells $p$ for dominance within the system.

Model equations including delay in the terms representing killing of virus-producing cells by CTL and in the stimulation of CTL:

In the basic delay model (1.11) we assume that killing of virus-producing cells by CTL is an instantaneous process. But in reality there is a latency period during this process. Thus we consider a delay in the factor in the terms representing killing of virus-producing cells by CTL and in the stimulation of CTL. We thus considered solutions of the following model equations

$$
\begin{aligned}
& \frac{d x}{d t}=\lambda-d x-\beta x y \\
& \frac{d y}{d t}=\beta x y-a y-p y(t-\tau) z \\
& \frac{d z}{d t}=k y(t-\tau)-b z
\end{aligned}
$$


Again in a more general model there would be a probability factor representing what happens to the activated virus-producing $\mathrm{T}$ cells between times $t-\tau$ and $t$, but we study the above approximation to this. One interpretation for this approximation is that it would be valid when the time delay is small. Similar models have been studied by other authors, see for example Wang et al. [39], Li et al. [22] and Yang et al. [43]. A similar idea is also used in other contexts by McCluskey [26], Bachar and Dorfmayr [1] and many other authors, see also our previous discussion.
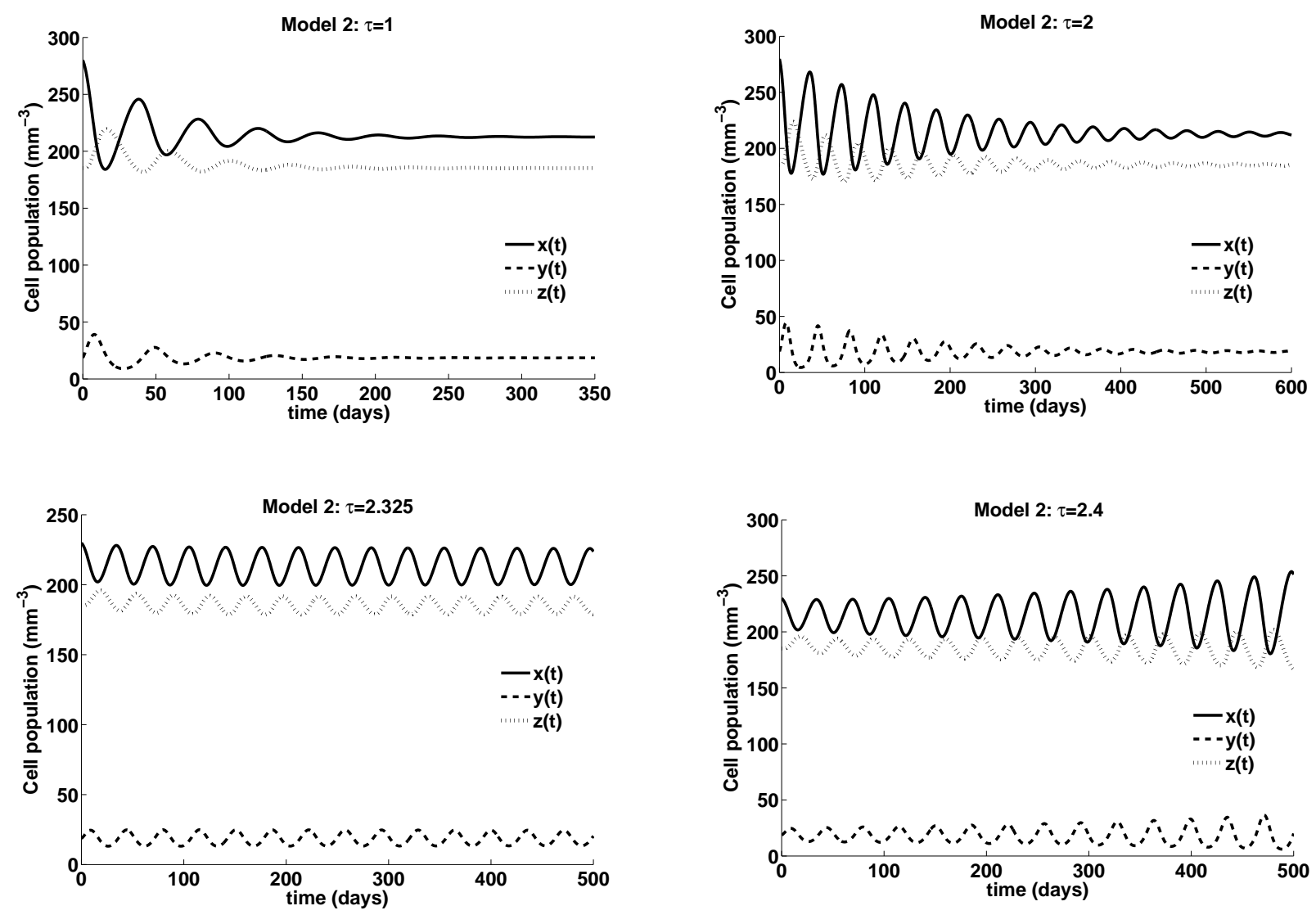

Figure 2: Time series solutions of model variables for different values of $\tau$ with perturbation. The model parameters are as in Table 1. $p=0.001 \mathrm{~mm}^{-3}$ day $^{-1}$.

In Figure 2 we again plot the time series solutions of the model variables corresponding to uninfected T cells, virus-producing T cells and CTL densities with initial perturbation of the uninfected $\mathrm{T}$ cells from the non-trivial equilibrium value, changing the value of the delay factor $\tau$. All of the other model parameters are chosen to assume their standard values as in Table 1. $p=0.001 \mathrm{~mm}^{-3}$ day $^{-1}$. Figures 2(a) (top left) and 2(b) (top right) have the same initial conditions as Figure 1(a), Figures 2(c) (bottom left) and 2(d) (bottom right) have initial conditions $x(\theta)=230.0, y(\theta)=18.5189$ in $(-\tau, 0]$ and $z(0)=185.1893$. Figure 2(a) has $\tau=1$ day, Figure 2(b) has $\tau=2$ days, Figure 2(c) has $\tau=2.325$ days and Figure $2(\mathrm{~d})$ has $\tau=2.4$ days.

Note that for both models our analytical results show that

$$
\frac{a}{\beta}<\frac{\lambda}{d}
$$


is a necessary and sufficient condition for there to be a unique equilibrium with virus present and for $\tau=0$ days this equilibrium is LAS. We find that increasing the delay increases the oscillation in the solution and also makes the oscillation persist for longer. In this respect the results of the model are qualitatively similar to the results of the model with time delay in the infection term (1.11). The increasing oscillatory trends in Figure 2(d) eventually reached regions of susceptible and virus-producing T cells and CTL where the approximate model was not valid. Increasing the time delay further did not lead to restabilization of the non-trivial equilibrium $E^{*}$, at least for realistic values of the time delay.

Other simulations (not presented) concentrated on the effect of changing the disease transmission term $\beta$. We found that as $\beta$ increases the amplitude of oscillations reduces. Also as $\beta$ increases the CTL population increases whereas the virus-producing and uninfected $\mathrm{T}$ cell populations decrease together.

Model equations including time delay in (i) in the process of infection of the healthy $\mathbf{T}$ cells, and (ii) the terms representing killing of virus-producing cells by CTL and in the stimulation of CTL together:

In this model we consider that delay exists in both (i) the process of infection of healthy $\mathrm{T}$ cells, and (ii) the terms representing killing of virus-producing cells by CTL and in the stimulation of CTL together. We thus consider the following model equations:

$$
\begin{aligned}
& \frac{d x}{d t}=\lambda-d x-\beta x\left(t-\tau_{1}\right) y\left(t-\tau_{1}\right), \\
& \frac{d y}{d t}=\beta x\left(t-\tau_{1}\right) y\left(t-\tau_{1}\right)-a y-p y\left(t-\tau_{2}\right) z, \\
& \frac{d z}{d t}=k y\left(t-\tau_{2}\right)-b z .
\end{aligned}
$$

The results are shown in Figure 3.

Figures 3(a)-3(c) (respectively top left, top right, bottom left) have the same initial conditions as Figures $2(\mathrm{c})$ and $2(\mathrm{~d})$. Figure $3(\mathrm{~d})$ (bottom right) has initial conditions $x(\theta)=$ 225.0, $y(\theta)=18.5189$ in $(-\tau, 0]$ and $z(0)=185.1893$. Figure $3\left(\right.$ a) has $\tau_{1}=3$ days and $\tau_{2}=$ 1 day, Figure 3(b) has $\tau_{1}=1$ day and $\tau_{2}=2$ days, Figure 3(c) has $\tau_{1}=3$ days and $\tau_{2}=$ 1.52 days and Figure $3(\mathrm{~d})$ has $\tau_{1}=4.1$ days and $\tau_{2}=1$ day.

Again we note that for all three models there is a unique non-trivial equilibrium if and only if

$$
\frac{a}{\beta}<\frac{\lambda}{d}
$$

and in the third model if $\tau_{1}=\tau_{2}=0$ then this equilibrium is LAS. We observe qualitatively that as either $\tau_{1}$ or $\tau_{2}$ increases the long-term dynamics tend to become more oscillatory. Again for the more oscillatory solutions the amplitude of the oscillations increased until they reached a region where the approximate model was not valid. Increasing $\tau_{1}$ and $\tau_{2}$ further did not seem to lead to restabilization of the non-trivial equilibrium $E^{*}$.

\section{Discussions and Conclusions:}

We have considered a mathematical model representing the response of an HIV-1 infected immune system to the CTL including delay in the disease transmission term and also two other terms. We have studied the first model with delay in only the disease transmission term both analytically and numerically, and the second model including delay in the 

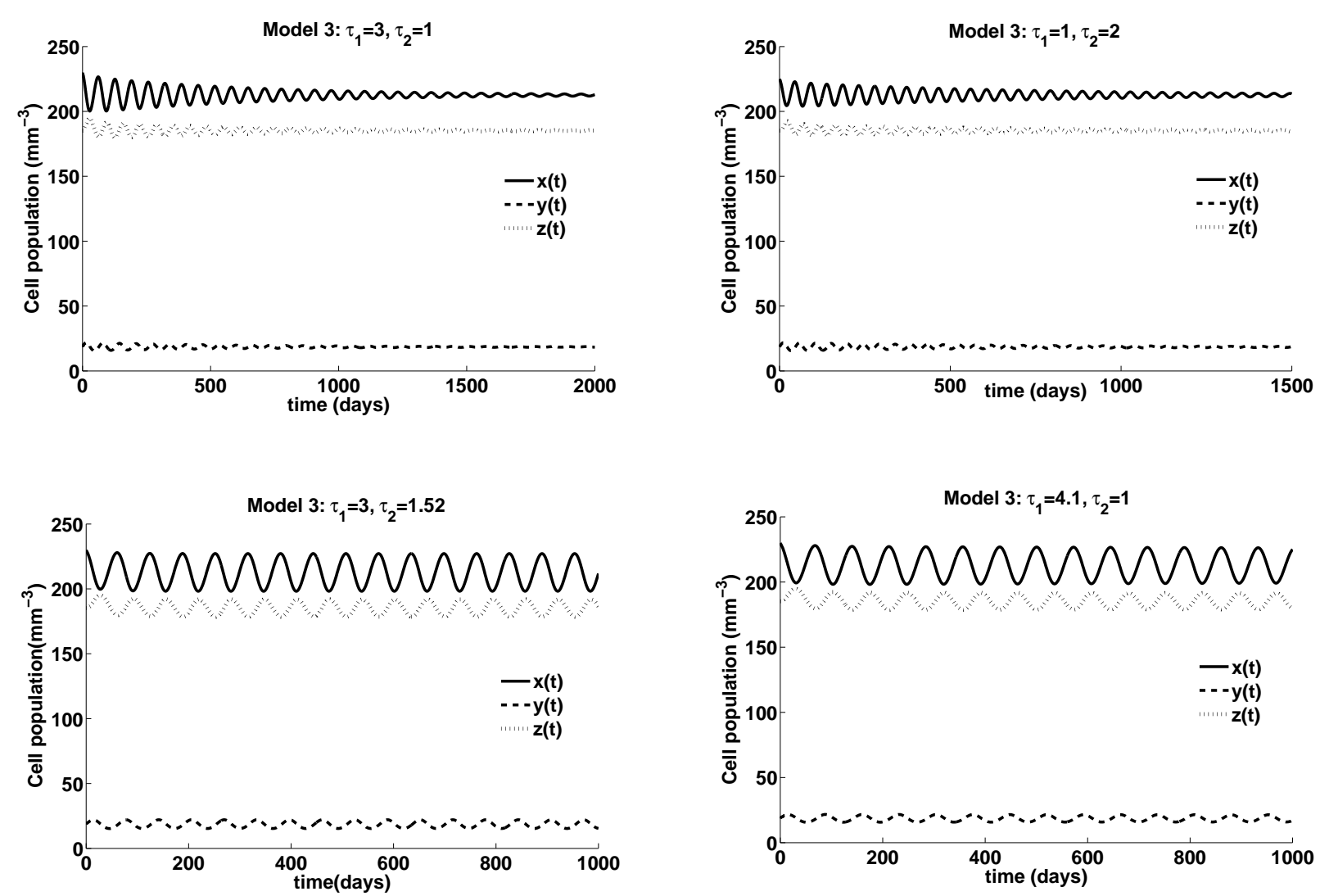

Figure 3: Time series solutions of model variables for different values of $\tau$ with perturbation. The model parameters are as in Table 1. $p=0.001 \mathrm{~mm}^{-3}$ day $^{-1}$.

terms representing killing of virus-producing cells by CTL, and in the stimulation of CTL numerically. We also studied the third model including all three types of delay numerically.

Our analytical results indicated that a time delay in the disease transmission term may cause the non-trivial equilibrium $E^{*}$ to become unstable as the time delay increases and we obtained conditions for this to happen. As the time delay increases further the non-trivial equilibrium may either always remain unstable, or it may cycle through an infinite sequence of regions alternately LAS and unstable. Again we found the conditions under which each of these possibilities would happen.

Numerical simulation of the model confirmed these results. We found that for realistic parameter values $E^{*}$ became unstable as the time delay increased but did not become stable again at higher time delay values. In the unstable region beyond the critical time delay the oscillatory solutions increased in amplitude until they reached a region where the approximate model was no longer valid. Our analysis also demonstrates that the time delay competes with the killing rate of infected cells by CTL $(p)$. This competition causes the parameters $\tau$ and $p$ to dominate the system depending on their respective numerical values. It was observed numerically that another parameter $k$ representing the rate of stimulation by CTL tends to dissipate the impact of instability caused by the delay in disease transmission. These simulations are not presented due to lack of space.

From discussion of the analytical solutions (for $\tau=0$ days) and the numerical solutions of the model equations (5.1) it is clear that delay in the killing rate of virus-producing $\mathrm{T}$ 
cells enhances oscillations in the model variables. Thus for example one can predict that if RTI drugs are applied so that $p$, the killing rate of infected T cells by CTL is $0.001 \mathrm{~mm}^{-3}$ day $^{-1}$ and $k$, the stimulation rate of CTL is 0.2 day $^{-1}$ and the delay is beneath a certain threshold value, then the RTI drugs drive the system to a steady state. However if this threshold delay value is exceeded then the level of infected $\mathrm{CD} 4^{+} \mathrm{T}$ cells will oscillate with increasing amplitude, along with the other variables in the system, until the system reaches a region where the approximate equations are no longer valid.
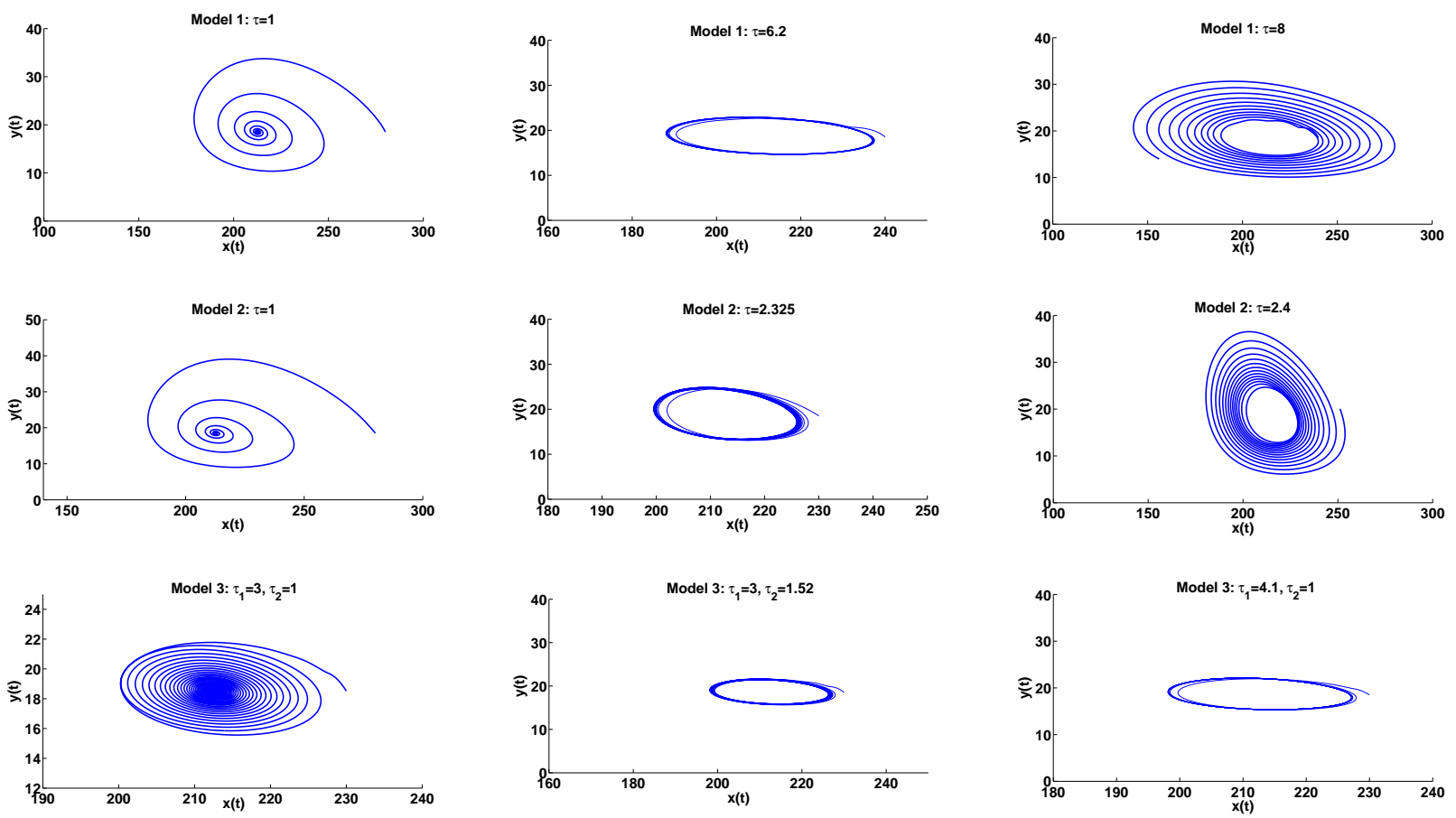

Figure 4: Time series solutions of model variables for different values of $\tau$ with perturbation. The model parameters are as in Table 1. $p=0.001 \mathrm{~mm}^{-3}$ day $^{-1}$.

Figure 4 shows phase plots of the delay induced system in the three different models: the model given by equations (1.11) (Figures 1(a), (c) and (d)), the model given by equations (5.1) (Figures 2(a), (c) and (d)) and the model given by equations (5.2) (Figures 3(a), (c) and (d)). In Figures 4(a) (top row left), (d) (middle row left) and (g) (bottom row left) the trajectories spiral inwards whereas in Figures 4(c) (top row right) and (f) (middle row right) the trajectories spiral outwards. In the top row of Figure 4 we see that there is a critical threshold value $\tau_{t h}=6.2$ days such that the system (1.11) is LAS for $\tau<\tau_{t h}$ and unstable for $\tau>\tau_{t h}$. These numerical results match our analytical predictions exactly. In the middle row of Figure 4 we see that the system (5.1) moves from local asymptotic stability to increasing oscillatory solutions, as $\tau$ passes through the approximate threshold value $\tau_{t h}=2.325$ days. The bottom row of Figure 4 suggests that the system (5.2) moves from being LAS for $\tau_{1}$ and $\tau_{2}$ small, to oscillatory solutions as $\tau_{1}$ and $\tau_{2}$ become larger. Again as we increase $\tau_{1}$ and $\tau_{2}$ further we observe oscillatory solutions of increasing amplitude diverging from the non-trivial equilibrium $E^{*}$.

Thus the conclusions of our analytical and numerical results can be briefly summarized by saying that increasing the time delay in the system causes increasing oscillations and instability in all of the models which we considered. This is a general conclusion for all the models for internal viral dynamics of HIV in the literature, both those which we discussed 
and those we did not. The models of Bonhoeffer et al. [2, 3], Dalal et al. [8] and Tuckwell and Wan [38] had no time delays and did not display oscillatory behaviour. The model of Wang et al. [39] included a time delay and displayed increasing oscillatory behaviour as the time delay increased. The model of Zhu and Zou [44] had a time delay but the equilibrium with infection present was always LAS. However the general principle remains that including a time delay will destabilize the system.

\section{Acknowledgement:}

We are grateful to the Government of India, Ministry of Science and Technology, Mathematical Science Office, the Indian National Science Academy and the Royal Society of Edinburgh, UK, for financial support.

\section{References}

[1] Bachar, M. and Dorfmayr, A., 2004. HIV treatment models with time delay. C. $R$. Biologies 327, 983-994.

[2] Bonhoeffer, S., Coffin, J. M. and Nowak, M. A., 1997. Human Immunodeficiency Virus drug therapy and virus load. J. Virol. 71, 3275-3278.

[3] Bonhoeffer, S., Shaw, G., May, R. and Nowak, M., 1997. Virus dynamics and drug therapy. Proc. Natl. Acad. Sci. 94, 6971-6976.

[4] Callaway, D. S. and Perelson, A. S., 2002. HIV-1 infection and low steady state viral loads. Bull. Math. Biol. 64, 29-64.

[5] Carmichael, A., Jin, X., Sissons, P. and Borysiewicz, L., 1993. Quantitative analysis of the human immunodeficiency virus type 1 (HIV-1)-specific cytotoxic T lymphocyte (CTL) response at different stages of HIV-1 infection: differential CTL response to HIV-1 and Epstein-Barr virus in late disease. J. Exp. Med. 177, 249-256.

[6] Coffin, J. M., 1995. HIV population dynamics in vivo: implications for genetic variation, pathogenesis, and therapy. Science 267, 483-489.

[7] Culshaw, R. V., Ruan, S. and Webb, G., 2003. A mathematical model of cell to cell spread of HIV-1 that includes a time delay. J. Math. Biol. 46, 425-444.

[8] Dalal, N., Greenhalgh, D. and Mao, X., 2009. Mathematical modelling of internal HIV dynamics. Disc. Cont. Dyn. Systems B 12, 305-321.

[9] Davenport, M. P., Ribeiro, R. M., Chao, D. L. and Perelson, S., 2004. Predicting the impact of a nonsterilizing vaccine against Human Immunodeficiency Virus. J. Virol. 78, 11340-11351.

[10] Erbe, L. H., Freedman, H. I. and Rao, V. S. H., 1986. Three species food chain models with mutual interference and time delays. Math. Biosci. 80, 57-80.

[11] Finzi, D. and Siliciano, R. F., 1998. Viral dynamics in HIV-1 infection. Cell 93, 665-671.

[12] Gopalsamy, K., 1992. Stability and Oscillations in Delay Differential Equations of Population Dynamics. Kluwer Academic, Dordecht-Boston-London. 
[13] Hasse, A. T., 1999. Population biology of HIV-1 infection: viral and CD4+ T cell demographics and dynamics in lymphatic tissues. Ann. Rev. Immunol. 17, 625-656.

[14] Herz, A. V., Bonhoeffer, S., Anderson, R. M., May, R. M. and Nowak, M. A., 1996. Viral dynamics in vivo; limitations on estimates of intracellular delay and virus decay. Proc. Natl. Acad. Sci. USA 93, 7247-7251.

[15] Ho, D. D., Neumann, A. U., Perelson, A. S., Chen, W., Leonard, J. M. and Markowitz, M., 1995. Rapid turnover of plasma virions and CD4 lymphocytes in HIV-1 infection. Nature 373(6510), 123-126.

[16] Kamina. A., Makuch, R. W. and Zhao, H., 2002. A stochastic modelling of early HIV-1 population dynamics. Math. Biosci. 170, 187-198.

[17] Kirschner, D. E. and Webb, G. F., 1996. A model of treatment strategy in the chemotherapy of AIDS. Bull. Math. Biol. 58, 367-390.

[18] Larder, B. A., Kemp, S. D. and Harrigan, P. R., 1995. Potential mechanism for sustained antiretroviral efficiency of AZT-3TC combination therapy. Science 269, 696-699.

[19] Layne, S. P., Spouge, J. L. and Dembo, M., 1989. Quantifying the infectivity of human immunodeficiency virus. Proc. Nat. Acad. Sci. USA 86, 4644-4468.

[20] Le Corfec, E., Le Pont, F., Tuckwell, H. C., Rouzioux, C. and Costagliola, D., 1999. Direct HIV testing in blood donations; variation of the yield with diction threshold and pool size. Transfusion 39, 1141-1144.

[21] Li, D. and Ma, W., 2007. Asymptotic properties of a HIV-1 infection model with time delay. J. Math. Anal. Appl. 335, 683-691.

[22] Li, Y., Xu, R., Li, Z. and Mao, S., 2011. Global dynamics of a delayed HIV-1 infection model with CTL immune response. Disc. Dyn. Nat. Soc. Article ID 673843, 13 pages doi:10.1155/2008/673843.

[23] MacDonald, M., 1978. Time Lags in Biological Models. Lecture Notes in Biomathematics. Vol. 27. Springer-Verlag, Berlin-Heidelberg-New York.

[24] Marsden, J. E. and McCracken, M., 1976. The Hopf Bifurcation and its Applications. Springer-Verlag, New York.

[25] May, R. M., 1974. Stability and Complexity in Model Ecosystems (Second Edition). Princeton University Press, Princeton.

[26] McCluskey, C. C., 2010. Complete global stability for an SIR epidemic model with delay - Distributed or discrete. Nonlin. Anal.: Real World Appl. 11, 55-59.

[27] Michie, C. A., McLean A., Alcock, C. and Beverley, P. C. L., 1992. Lifespan of human lymphocyte subsets defined by CD45 isoforms. Nature 360, 264-265.

[28] Murray, J. M., Kaufmann, G., Kelleher, A. D. and Cooper, D. A., 1998. A model of primary HIV infection. Math. Biosci. 154, 57-85. 
[29] Nowak, M. A. and May, R. M., 1993. AIDS pathogenesis: Mathematical models of HIV and SIV infections. AIDS 7, S3-S18.

[30] Perelson, A. S., Neumann, A. U., Markowitz, M., Leonard, J. M. and Ho, D. D., 1996. HIV 1 dynamics in vivo: virion clearance rate, infected cell life span, and viral generation time. Science 271, 1582-1586.

[31] Perelson, A. S., Kirschner, D. E and De Boer, R., 1993. Dynamics of HIV infection of $\mathrm{CD}^{+}$T cells. Math. Biosci. 114, 81-125.

[32] Pierson, T., McArthur, J. and Siliciano, R. F., 2000. Reservoirs for HIV-1: mechanisms for viral persistence in the presence of antiviral immune responses and antiretroviral therapy. Ann. Rev. Immunol. 18, 665-708.

[33] Phillips, A. N., 1996. Reduction of HIV concentration during acute infection: independence from a specific immune response. Science 271, 497-499.

[34] Rosenberg, Y. and Janossy, G., 1999. The importance of lymphocyte trafficking in regulating blood lymphocyte levels during HIV and SIV infections. Seminars Immunol. $11,139-154$.

[35] Shi, X., Zhou, X. and Song, X., 2010. Dynamical behaviour of a delay virus dynamics model with CTL immune response. Nonlin. Anal.: Real World Appl. 11, 1795-1809.

[36] Stafford, M. A., Corey, L., Cao, Y., Daar, E. S., Ho, D. D. and Perelson, A.S., 2000. Modelling plasma virus concentration during primary HIV infection. J. Theor. Biol. 203, 285-301.

[37] Tan, W. Y. and Wu, H., 1998. Stochastic modelling of the dynamics of CD4 T cell infection by HIV and some Monte Carlo studies. Math. Biosci. 147, 173-205.

[38] Tuckwell, H. and Wan, F., 2000. Nature of equilibria and effects of drug treatments in some simple viral population dynamical models. IMA J. Math. Appl. Med. Biol. 17, 311-327.

[39] Wang, K., Wang, W., Pang, H. and Liu, X., 2007. Complex dynamic behavior in a viral model with delayed immune response. Physica D 226, 197-208.

[40] Wodarz, D., May, R. M. and Nowak, M. A., 2000. The role of antigen-independent persistence of memory cytotoxic T lymphocytes. Internat. Immunol. 12, 467-477.

[41] Wodarz, D. and Nowak, M. A., 1999. Specific therapy regimes could lead to long-term immunological control to HIV. Proc. Natl. Acad. Sci. USA 96, 14464-14469.

[42] Yang, J., Wang, X. and Zhang, F., 2008. A differential equation model of HIV infection of $\mathrm{CD}^{+} \mathrm{T}$ cells with delay. Disc. Dyn. Nat. Soc. Article ID 903678, 16 pages doi:10.1155/2008/903678.

[43] Yang, J.-Y., Wang, X.-Y. and Li, X.-Z., 2011. Hopf bifurcation for a model of HIV infection of CD4+ T cells with virus released delay. Disc. Dyn. Nat. Soc. Article ID 649650, 24 pages doi:10.1155/2011/649650. 
[44] Zhu, H. and Zou, X., 2008. Impact of delays and virus production on HIV-1 dynamics. Math. Med. Biol. 25, 99-112.

[45] Zurakowski, R. and Teel, A. R., 2006. A model predictive control based scheduling method for HIV therapy. J. Theoret. Biol. 238, 368-382. 